\title{
Ability Training in Educational Model of Feasibility Study Studio
}

\author{
Hongxiong Yang \\ School of Management, Tianjin University of Technology, Tianjin, CHINA \\ Yifan Pan \\ School of Design \& Environment, National University of Singapore, SINGAPORE \\ Jinqing $\mathrm{HaO}$ \\ Library, Nankai University, Tianjin, CHINA
}

Received 12 June 2017 - Revised 26 August 2017 - Accepted 25 September 2017

\begin{abstract}
This paper proposed a training system in the educational model of feasibility study studio considering feedback mechanism based on the learning process and knowledge bank. System dynamic theory was used to analyze information feedback between undergraduates and teachers as well as among undergraduates. Positive feedback stimulates and negative feedback inhibits practical ability improvement in the studio educational model. An initial function with dynamic features was established according to the feedback mechanism, the function's differential equation was solved to get the general solution. An " $\mathrm{S}$ " curve demonstrates the trend of practical ability training performance in educational model of feasibility study studio. The formation process of the "S" curve was explained. At last, some methods running the studio were proposed to elongate positive feedback and improve practical ability training performance in the educational model. This paper established the ability training pattern educational model of feasibility study studio and realized the integration of modules and platforms with theoretical knowledge as the inputs and practical abilities as the outputs.
\end{abstract}

Keywords: the educational model of feasibility study studio (EMFSS), ability training system, positive feedback, negative feedback, module, platform

\section{INTRODUCTION}

The practice teaching in university education gradually grows out of symbolic participation towards full participation. The educational model of studio changes the traditional method into a practice teaching mode, which is from teachers being the main component, teaching content being the core and practice activities being the carriers to undergraduates being the main component, materialization of knowledge and skills being the core content and studios being the carriers (Rodriguez et al., 2016). With undergraduates being the main component, building a platform for smart communication is a must (Rodriguez et al., 2016; O'Dwyer and Childs, 2017). With materialization of knowledge and skills being the core content, building a comprehensive studio knowledge bank is a must. With studios being the carriers, a feedback mechanism on the knowledge and information in studio training system is needed so that the undergraduates' learning processes are macro controllable and micro adjustable (Alizadeh et al., 2017; Tsai, 2017). The feedback mechanism share, integrate and correct the knowledge and information in studio. The educational model of studio is the incubator for practical ability of undergraduates (Fasli and Hassanpour, 2017). Undergraduates graduated from this studio should be equipped with good communication skills, team cooperation skills, solid basic theories, strong technical application abilities, broad

(C) Authors. Terms and conditions of Creative Commons Attribution 4.0 International (CC BY 4.0) apply. Correspondence: Yifan Pan, School of Design \& Environment, National University of Singapore, SINGAPORE. 


\section{Contribution of this paper to the literature}

- This paper builds the guarantee system for the ability training of studio, which has surpassed the situation that much existing literature mainly discusses the process of capability improvement.

- Taking feedback mechanism as the core, this paper analyzes the mechanism of improving the competence in studio education model.

knowledge and good all-around knowledge ability to meet the market requirements. Therefore, appointing individuals to accomplish studio tasks within a group-based framework creates a combination of strategies that can achieve both individual-based and group-based objectives (Soliman AM, 2017).

The engineering education is changing towards educating critical thinkers who are equipped with risktaking abilities (Fasli and Hassanpour, 2017; Lin et al., 2017; Hsiao, 2017). The ability of feasibility study is an important skill to be mastered for engineering undergraduates. These skills will be developed in educational model of feasibility study studio (EMFSS). The undergraduates learn to compile feasibility reports and obtain investing \& finance skills. EMFSS transfers tasks of solving problem to undergraduates. The team members of studio discuss how to obtain information, how to assemble content and how to use technique (Greer K, 2016). The discussion is supported by a discussion platform based on relevant knowledge bank. EMFSS training system is made up of feasibility study communication platform and knowledge bank, which provides the workplace for the feedback mechanism. The system solves the connection problem between knowledge and skill during studio practice teaching, pushes innovation in practice teaching, and enables seamless connection between university teaching and market requirements. (Liao XM, 2017; Huang et al.,2016).

\section{BUILD THE EMFSS TRAINING SYSTEM}

\section{Implementation Process of EMFSS}

In EMFSS, the fact that undergraduates are encouraged to take their own value positions and to incorporate them in a feasibility study report to reflect this, meant that they rehearsed the abstraction and reinsertion of values into their own works of the specific project site and context. This introduces skills applicable in any site or social context, so that the disciplined expression of meaning, which is what value is, may be a feasibility study technique potentially applicable anywhere in the world. (Chiaradia et al., 2017)

EMFSS trains undergraduate calculation and analysis abilities on various parameters of the feasibility study. It also trains assessment ability and decision ability on a project as a whole and overall. The training process takes into account economic, social, natural and policy factors, which enable members to judge project feasibility from a long-term and objective perspective. Table 1 shows training process, illustrates the task and the task sequences in EMFSS.

\section{Training System of EMFSS}

The training system of EMFSS includes three platforms and three modules, as shown in Figure 1. The three platforms are knowledge bank platform to realize abilities in EMFSS, cases sharing platform for ability realization of EMFSS, and communication platform for problems during feasibility report making. Three platforms serve feedback site, feedback opportunity and feedback medium in the studio while three modules serve feedback purpose. The three modules of feasibility study include ability module, tasks module and business module. The training system of EMFSS shows the logical relationship between knowledge, ability, tasks and business from shallow to deep, from simple to complex, from low level to high level, from left to the right and from bottom to top. Every stage and every step in Table 1 can correspond to a node in Figure 1. Knowledge and skills can accumulate through node modules and platform, and expand to a higher level. (Wang et al.,2016; Yan et al.,2016) 


\section{ESTABLISH THE FEEDBACK MECHANISM FOR TRAINING SYSTEM OF EMFSS}

The training system of EMFSS is composed of two components, namely the teachers and undergraduates, upon which either positive or negative feedback will emerge based on the interplay and influence each other. The studio education needs to create feedback platforms, it highlight the need to create opportunities for undergraduates to engage with project stakeholders, engage in dialogue with project demanders and appreciate the value of local knowledge and input in project report. The feedback platforms encourage undergraduates to explore creative problem-solving in practice through design proposals based on negotiation, resource allocation, hybridity, unconventional configurations and attention to small, often ignored, spaces. (Loukaitou-Sideris and Vinit Mukhija, 2016).

\section{Peer Feedback and Teacher Feedback}

Peer feedback based on the three platforms are carried out in team and inter-team to discuss on knowledge points in different banks, case details, and difficulties in making feasibility reports. Team members learn synchronously, they can learn from peers by avoiding others' mistakes, so peer feedback can improve undergraduates' ability to implement the knowledge. Repetitive peer feedback can also stimulate more feedback response of peer. This communication feedback process can motivate undergraduates to use textbook knowledge as flexible as possible and strengthen their practice abilities of the feasibility study, this effect rather than passively receiving teacher instructions. Peer feedback provides members with real clients, readers and judges, which increase undergraduates' awareness to check clients' needs and requirements during report making and helps them to develop from "do it to fulfil teachers' requirements" to "do it to fulfil clients' feasibility study needs." Establishing this counseling awareness can help undergraduates to seriously consider clients' theoretical and real needs, to stand in clients' shoes and build a rigorous analysis attitude which combines qualitative and quantitative analysis, make correct project decisions. As a kind of cooperation between undergraduates, peer feedback motivates undergraduates to make the feasibility study report through discussion and negotiation. It sharpens undergraduates' oral and written communication, writing, critical thinking and self-learning skills (Kilinc et al., 2017; $\mathrm{Wu}, 2012)$.

Teacher feedback bases also on three platforms. Such feedback dispels the difficulties of undergraduates encountering during making the report, by written and oral mode in class. Teacher also suggests that the feedback framework of scope, context, process and practice can help guide work of feasibility study responses to project environment. To eliminate undergraduates' fallacy that teacher feedback is more authoritative and comprehensive, teacher feedback serves as a complementary method to peer feedback in running the studio.

Peer feedback and teacher feedback are not interchangeable; each of them is a part of the feedback mechanism and should integrate effectively. Regarding feedback method and content, vague and excessive feedbacks can exist in peer feedback. Vague feedback means undergraduates give overly summarized feedbacks commenting on peer's feasibility report. They do not point the keys of problems or do not offer specific suggestions. On the contrary, some able undergraduates may give excessive feedbacks, means the feedbacks are so detailed that almost all revision has been done, thus depriving others of the opportunities to learn and improve. Excessive teacher feedback should also be avoided not to impair undergraduates' enthusiasm to learn by themselves.

\section{Positive and Negative Dynamic Feedback Mechanism in EMFSS}

\section{The roles of feedback mechanism}

The proper connection of modules and platform in the training system in EMFSS motivate and give confidence to members to cooperate with each other. Peer feedback stimulates interaction and communication between undergraduates; teacher feedback guides undergraduates to the accurate searching way of solving the problem. After multiple feedback stages, invisible as well as visible knowledge is shared and transmitted, learning performance explodes exponentially, and members' practical abilities increase gradually in EMFSS. 
Positive feedback cannot infinitely expand; it is inhibited by negative feedback. Some members out of their reasons are not willing to share knowledge and information with others. Other undergraduates may waste each other's time by discussing and communicating excessively. Excessive teacher feedback can tire and bore undergraduates or make them unconfident about their abilities to practice feasibility study. All these can lower the growing speed of performance of practical ability in EMFSS and lead to negative feedback cycle.

\section{The dynamic performance model for feedback mechanism of shaping practical ability in EMFSS}

Here is a dynamic model based on system dynamic theory, it is proposed to analyze the feedback's influence process. The model takes feedback as function variable and studies the influence on feedback's performance of shaping practical ability in EMFSS as it changes. The initial performance of shaping practical ability in EMFSS is relatively stable, it doesn't change over time or the change is negligible. Moderate feedback increases knowledge and skill, while too much or too less feedback inhibits and slows down the increase in shaping practical ability performance.

\section{Set the initial function as}

Take KM as the initial performance value of shaping practical ability in EMFSS, CM as performance added value of feedback to problems in making feasibility study reports, $\mathrm{O}$ as performance reduction value due to improper feedback of shaping ability in EMFSS, PM as the performance of shaping practical ability in EMFSS. PM changes as $\mathrm{CM}$ or $\mathrm{O}$ changes. $\mathrm{K} 1$ and $\mathrm{K} 2$ are two influencing factors of time on the initial value of performance of shaping practical ability in EMFSS, both factors are larger than 0. The Equations are as follows:

According to Function 1, get Function 5: Function 6:

Both sides of function 5 divided by $d_{t}$, then substitute the variables with Functions (2), (3) and (4), get

Then get the following differential equations:

General solution to Function 7 is as follows:

In which $\mathrm{C}_{1}$ is any constant.

Solve the differential equation and get the general solution, get the growth curve for the performance of shaping practical ability in EMFSS under the positive and negative influence of the feedback, illustrated in Figure 2. The initial value of performance of shaping practical ability in EMFSS is $\mathrm{C}_{1}+\mathrm{k}_{2}$. At this stage, an orderly structure of the practical ability in EMFSS is forming, but with low efficiency, the growth trend of practical ability efficiency is not obvious. After the positive and negative feedback's effect on EMFSS, PM's initial value is slightly higher than $\mathrm{C}_{1}+\mathrm{k}_{2}$. After a time span of $[0, \mathrm{t}]$, factors inhibiting practical ability shaping increase gradually, and the effect of negative feedback becomes larger than positive feedback. The change rate of PM decreases gradually until 0 , and the $P M$ value shows as a typical "S" curve.

According to the above analysis, teachers and undergraduates should pay attention to the method and extent of problem feedback during the process of making feasibility report. The people who provide feedback should provide more guidance, enlightenment, and paths but not help those having difficulties to accomplish directly. Thus the growth stage of $[0, t]$ on the S-curve can be extended as long as possible and enter the next exponential growth cycle as soon as possible when the PM performance turns stable.

\section{CONCLUSION}

The research demonstrates the connection pattern between the three modules and three platforms in the training system of EMFSS. It uses system dynamic theory to explain the positive and negative feedback mechanism 
between undergraduates and teachers and among undergraduates. The research discovered the feedbacks could stimulate as well as inhibit practical ability improvement in EMFSS. At the same time, this research establishes an initial function with dynamic features according to the feedback mechanism. By solving the function's differential equation and achieving the general solution, the performance of practical ability training in EMFSS demonstrates an "S" curve. The formation process of the "S" curve is explained by solving the equation as well. At last, specific strategies to elongate positive feedback thus improves the performance of practical ability training in the studio were proposed. This paper established the ability training pattern in EMFSS and realized the integration of modules and platforms in which theoretical knowledge are the inputs, and practical abilities are the outputs.

This paper has suggested that EMFSS is both defined by the necessity of project construction and helps define it, and should be an active focus for engineering major pedagogy. It establishes the educational idea to take undergraduates as the protagonist based on the feedback mechanism of three modules and three platforms. The idea absorbs various elements such as interactive study and practical teaching in a flexibly way, takes project needs and social needs as the core content seriously, hands out tasks such as solving project construction scale, financing, finance structure, finance efficiency to undergraduates to analyze and proof. The undergraduates in EMFSS improve their feasibility study skills by professional instructions and feedback mechanism.

\section{ACKNOWLEDGEMENTS}

This work is part of the YB14-17 (Research on feasibility analysis ability based on modular connection _ studio perspective). The authors would like to gratefully acknowledge Tianjin University of Technology for financial support through its teaching reform project.

\section{REFERENCES}

Alizadeh, T., Tomerini, D., \& Colbran, S. (2017). Teaching planning studios: an online assessment task to enhance the first year experience. Journal of Planning Education and Research, 37(2), 234-245. doi:10.1177/0739456X16647162

Chiaradia, A. J. F., Sieh, L., \& Plimmer, F. (2017). Values in urban design: a design studio teaching approach. Design Studies, 49(C), 66-100. doi:10.1016/j.destud.2016.10.002

Fasli, M., \& Hassanpour, B. (2017). Rotational critique system as a method of culture change in an architecture design studio: urban design studio as case study. Innovations in Education and Teaching International, 54(3), 194-205. doi:10.1080/14703297.2016.1174142

Gingerich, C. (2016). Possibilities, opportunities and entrepreneurship: taking creativity beyond the studio. Mtna E-journal, November 2016, 17-19.

Greer, K. (2016). Undergraduate studio art information use a multi-school citation analysis. Journal of the Art Libraries Society of North America, 35(2), 230-240. doi:10.1086/688725

Hsiao, H.-S., Chen, J.-C., Hong, J.-C., Chen, P.-H., Lu, C.-C., \& Chen, S. Y. (2017). A five-stage Predictionobservation-explanation inquiry-based Learning Model to improve Students' Learning Performance in Science Courses. Eurasia Journal of Mathematics Science and Technology Education, 13(7), 3393-3416. doi:10.12973/eurasia.2017.00735a

Huang, Q. L., Li, B. M., \& Ren, Y. Q. (2016). Empirical study on depth of online discussion in teachers workshops _ a case analysis of teachers workshop of ICT competency enhancement project. E-Education Research, 37(12), 121-128. doi:10.13811/j.cnki.eer.2016.12.018

Ji, Y. C. (2017). An investigation of design studio performance in relation to creativity, spatial ability, and visual cognitive style. Thinking Skills and Creativity, 23, 67-78. doi:10.1016/j.tsc.2016.11.006

Kilinc, A., Demiral, U., \& Kartal, T. (2017). Resistance to dialogic discourse in ssi teaching: the effects of an argumentation-based workshop, teaching practicum, and induction on a preservice science teacher. Journal of Research in Science Teaching, 54(6), 764-789. doi:10.1002/tea.21385 
Knaub, A. V., Foote, K. T., Henderson, C., Dancy, M., \& Beichner, R. J. (2016). Get a room: the role of classroom space in sustained implementation of studio style instruction. International Journal of STEM Education, December 2016, 3(8), 1-22. doi:10.1186/s40594-016-0042-3

Lin, M. T.-Y., Wang, J.-S., Kuo, H.-M., \& Luo, Y. (2017) A Study on the Effect of Virtual Reality 3D Exploratory Education on Students' Creativity and Leadership. Eurasia Journal of Mathematics Science and Technology Education, 13(7), 3151-3161. doi:10.12973/eurasia.2017.00709a

Liao, X. M. (2017). Promoting autonomic learning of undergraduate through learning community model _ based on the studio of learning guidance in Wenhua College. China Higher Education Research, (1), 91-94. doi:10.16298/j.cnki.1004-3667.2017.01.18

Loukaitou-Sideris, A., \& Vinit Mukhija, V. (2016). Responding to informality through urban design studio pedagogy. Journal of Urban Design, 21(5), 577-595. doi:10.1080/13574809.2015.1071650

O'Dwyer, A., \& Childs, P. E. (2017). Who says Organic Chemistry is Difficult? Exploring Perspectives and Perceptions. Eurasia Journal of Mathematics Science and Technology Education, 13(7), 3599-3620. doi:10.12973/eurasia.2017.00748a

Puccinelli, E. (2016). Thoughts and tools for team building in the studio. Journal of Singing, 73(2), 215-218.

Rodriguez, C., Hudson, R., \& Niblock, C. (2016). Collaborative learning in architectural education: Benefits of combining conventional studio, virtual design studio and live projects. British Journal of Educational Technology, 1-17. doi:10.1111/bjet.12535

Soliman, A. M. (2017). Appropriate teaching and learning strategies for the architectural design process in pedagogic design studios. Frontiers of Architectural Research, 6(2), 204-217. doi:10.1016/j.foar.2017.03.002

Tsai, F. (2017) An Investigation of Gender Differences in a Game-based Learning Environment with Different Game Modes. Eurasia Journal of Mathematics Science and Technology Education, 13(7), 3209-3226. doi:10.12973/eurasia.2017.00713a

Wang, X. C., Liao, J. S., Wang, X. M., \& You, Z. W. (2016). Inspiring form of group innovation in the teaching of design workshops. Journal of Beijing University of Posts and Telecommunications (Social Sciences Edition), 18(5), 102-108.

Wu, Y., Li, X. Q., \& Xia, D. (2012). The research on the feedback mechanism of communication management in knowledge innovation process in research team. Science $\mathcal{E}$ Technology Progress and Policy, 29(1), 7-10.

Yan, L., Chen, Y. W., \& Deng, J. J. (2016). Research on implementation mode of studio practice teaching based on question standards. Modern University Education, (5), 94-103.

\section{http://www.ejmste.com}

